\title{
LIMITS OF PHOTOSYNTHESIS IN EXTRASOLAR PLANETARY SYSTEMS FOR EARTH-LIKE PLANETS
}

\author{
S. Franck ${ }^{1}$, W. von Bloh ${ }^{1}$, C. Bounama ${ }^{1}$, M. Steffen ${ }^{2}$, D. Schönberner ${ }^{2}$, and H. J. Schellnhuber ${ }^{1}$ \\ ${ }^{1}$ Potsdam Institute for Climate Impact Research, PF 601203, 14412 Potsdam, Germany \\ ${ }^{2}$ Astrophysical Institute Potsdam, An der Sternwarte 16, 14482 Potsdam, Germany
}

\begin{abstract}
We present a general modeling scheme for investigating the possibility of photosynthesis-based life on extrasolar planets. The scheme focuses on the identification of the habitable zone in main-sequence-star planetary systems with planets of Earth mass and size. Our definition of habitability is based on the longterm possibility of photosynthetic biomass production as a function of mean planetary surface temperature and atmospheric $\mathrm{CO}_{2}$-content. All the astrophysical, climatological, biogeochemical, and geodynamic key processes involved in the generation of photosynthesis-driven life conditions are taken into account. Implicitly, a co-genetic origin of the central star and the orbiting planet is assumed. The numerical solution of an advanced geodynamic model yields realistic look-up diagrams for determining the limits of photosynthesis in extrasolar planetary systems, assuming minimum $\mathrm{CO}_{2}$ levels set by the demand of $\mathrm{C}_{4}$ photosynthesis.
\end{abstract}

\section{INTRODUCTION}

The question of extraterrestrial life is one of the most basic ones in the history of science. For many years scientists have been very attracted to the idea of the possibility of life on other planets in our solar system and in other planetary systems. On the basis of our knowledge about life existing on Earth we assume that extraterrestrial life has a carbon-based structure and needs liquid water. These are the main assumptions for limiting photosynthesis in extrasolar planetary systems. In this sense a habitable planet is defined as a planet capable of supporting such life. The habitable zone (HZ) of distances between a main sequence star and an Earth-like planet is roughly defined as the range of mean orbital radii which imply moderate planetary surface temperatures suitable for the development and subsistence of photosynthesis based life forms. The conventional definition of the $\mathrm{HZ}$ is the region in space around a star where liquid water can exist on a planet's surface (e.g. Doyle, 1996). The HZ concept was introduced by Huang (1959, 1960) and extended by Shklovskii and Sagan (1966), respectively. Hart (1978, 1979) calculated the HZ for G2 stars like our Sun to be amazingly narrow (between $0.958 \mathrm{AU}$ and $1.004 \mathrm{AU}$ ). Through the inclusion of the negative feedback between atmospheric $\mathrm{CO}_{2}$ partial pressure and mean global temperature via the carbonate-silicate cycle by Kasting et al. (1988) the outer boundary of the HZ was remarkably extended. Later on the $\mathrm{HZ}$ approach was modified to other classes of main sequence stars (Kasting et al., 1993; Kasting, 1997; Williams, 1998). A thorough state-of-the-art overview is provided by Doyle (1996). Recent studies by Franck et al. (2000a, 2000b) have generated a rather comprehensive 
characterization of the possibility of photosynthetic biomass production. Thus not only the availability of liquid water on a planetary surface is essential but also the suitability of $\mathrm{CO}_{2}$ partial pressures.

\section{MODEL DISCRIPTION}

Our modeling approach is based on a simulation of the coupling between increasing central star luminosity, silicate rock weathering, and the global energy balance (Caldeira and Kasting, 1992; Franck et al., 2000b). As a direct result, the partial pressure of atmospheric carbon dioxide $P_{a t m}$ and the biological productivity $\Pi$ can be estimated as functions of time $t$ throughout planetary past and future.

The luminosity evolution of a main-sequence central star in the mass range between 0.8 and 2.5 solar masses $\left(M_{s}\right)$ was calculated by polynomial fitting of detailed stellar evolution models and presented in the form of a Hertzsprung-Russell diagram (Franck et al., 2000b). For stellar masses between 0.2 and $0.8 \mathrm{M}_{\mathrm{s}}$ the luminosity $L$ can be extrapolated with the help of the luminosity-mass relation, $L \sim M^{3.88}$ (Kippenhahn and Weigert, 1990).

The global energy balance governing the climate of candidate planets is based on a formula given by Williams (1998):

$$
\frac{L}{4 \pi R^{2}}\left[1-a\left(T_{\text {surf }}, T_{e f f}, P_{a t m}\right)\right]=4 I\left(T_{\text {surf }}, P_{a t m}\right)
$$

where $R$ is the distance from the central star, $a$ the planetary albedo, $T_{\text {surf }}$ the mean surface temperature of the planet, $T_{\text {eff }}$ the stellar radiation temperature, and $I$ the outgoing infrared flux.

The weathering process has the potential to stabilize the planet's surface temperature by a negative feedback that is strongly modulated by the biosphere (Walker et al., 1981; Schwartzman and Volk, 1989; Schneider and Boston, 1991). The total process of weathering embraces first the reaction of silicate minerals with carbon dioxide, second the transport of weathering products, and third the deposition of carbonate minerals in sediments. Following Walker et al. (1981) and Caldeira and Kasting (1992), the global mean silicate-rock weathering rate $F_{w r}$ can be formulated via the following equation:

$$
\frac{F_{w r}}{F_{w r, 0}}=\left(\frac{a_{H^{+}}}{a_{H^{+}, 0}}\right)^{0.5} \exp \left(\frac{T_{\text {surf }}-T_{\text {surf }, 0}}{13.7 \mathrm{~K}}\right) \text {. }
$$

Here the pre-factor outlines the role of $\mathrm{CO}_{2}$ concentration in the soil, $P_{\text {soil }} . a_{H^{+}}$is the activity of $\mathrm{H}^{+}$in fresh soil-water. The quantities $F_{w r, 0}, a_{H^{+}, 0}$, and $T_{s u r f, 0}$ are the present-day values for the weathering rate, the $\mathrm{H}^{+}$activity, and the surface temperature, respectively.

Following Volk (1987), $P_{\text {soil }}$ can be assumed to be linearly related to the terrestrial biological productivity $\Pi$, defined as biomass production per unit time and per unit area, and to the atmospheric $\mathrm{CO}_{2}$ partial pressure $P_{a t m}$ :

$$
\frac{P_{\text {soil }}}{P_{\text {soil }, 0}}=\frac{\Pi}{\Pi_{0}}\left(1-\frac{P_{a t m, 0}}{P_{\text {soil }, 0}}\right)+\frac{P_{a t m}}{P_{\text {soil }, 0}},
$$

where $P_{\text {soil, },}, \Pi_{0}$, and $P_{a t m, 0}$ are again present-day values.

The main role of the biosphere in the context of our model is to enrich $P_{\text {soil }}$, relative to the atmospheric value $P_{a t m}$, in proportion to the biological productivity $\Pi$. $\Pi$ is considered to be a function of temperature and $\mathrm{CO}_{2}$ partial pressure in the atmosphere only: 


$$
\frac{\Pi}{\Pi_{\max }}=\left(1-\left(\frac{T_{\mathrm{surf}}-50^{\circ} \mathrm{C}}{50^{\circ} \mathrm{C}}\right)^{2}\right)\left(\frac{P_{\mathrm{atm}}-P_{\mathrm{min}}}{P_{1 / 2}+\left(P_{\mathrm{atm}}-P_{\min }\right)}\right) .
$$

Here $\Pi_{\max }$ denotes the maximum productivity, which is assumed to amount to twice the present value $\Pi_{0}$ (Volk, 1987). $P_{1 / 2}+P_{\min }$ is the value at which the pressure-dependent factor is equal to $1 / 2$, and $P_{\min }$ is fixed at $10^{-5}$ bar, the presumed minimum value for $\mathrm{C}_{4}$-photosynthesis (Pearcy and Ehleringer, 1984; Larcher, 1995). The evolution of teh biosphere and its adaption to even lower $\mathrm{CO}_{2}$ partial pressures are not taken into account in our model. The evolution of $\mathrm{CO}_{2}$ concentrating mechanisms in $\mathrm{C}_{4}$ photosynthesis in the past (some time after the end of the late Cretaceous) shows that this is an open possibilty for the future. For a given $P_{\text {atm }}$, Eq. 4 yields maximum productivity at $T_{\text {surf }}=50^{\circ} \mathrm{C}$ and zero productivity for $T_{\text {surf }} \leq 0^{\circ} \mathrm{C}$ and $T_{\text {surf }} \geq 100^{\circ} \mathrm{C}$. At this point we should emphasize that all calculations are done for a planet with Earth mass and size, and the sameoactive heating in its interior.

In this way, the $\mathrm{HZ}$ is defined as the spatial domain around a central star where the planetary surface temperature stays between $0^{\circ} \mathrm{C}$ and $100^{\circ} \mathrm{C}$ and where the atmospheric $\mathrm{CO}_{2}$ partial pressure is higher than $10^{-5}$ bar to allow photosynthesis. This is equivalent to a nonvanishing biological productivity, i.e. $\Pi>0$ :

$$
H Z:=\left\{R \mid \Pi\left(P_{a t m}(R, t), T(R, t)\right)>0\right\} .
$$

Our model has also an upper limit for atmospheric $\mathrm{CO}_{2}$ at 10 bar (Kasting and Ackerman, 1986; Tajika and Matsui, 1992).

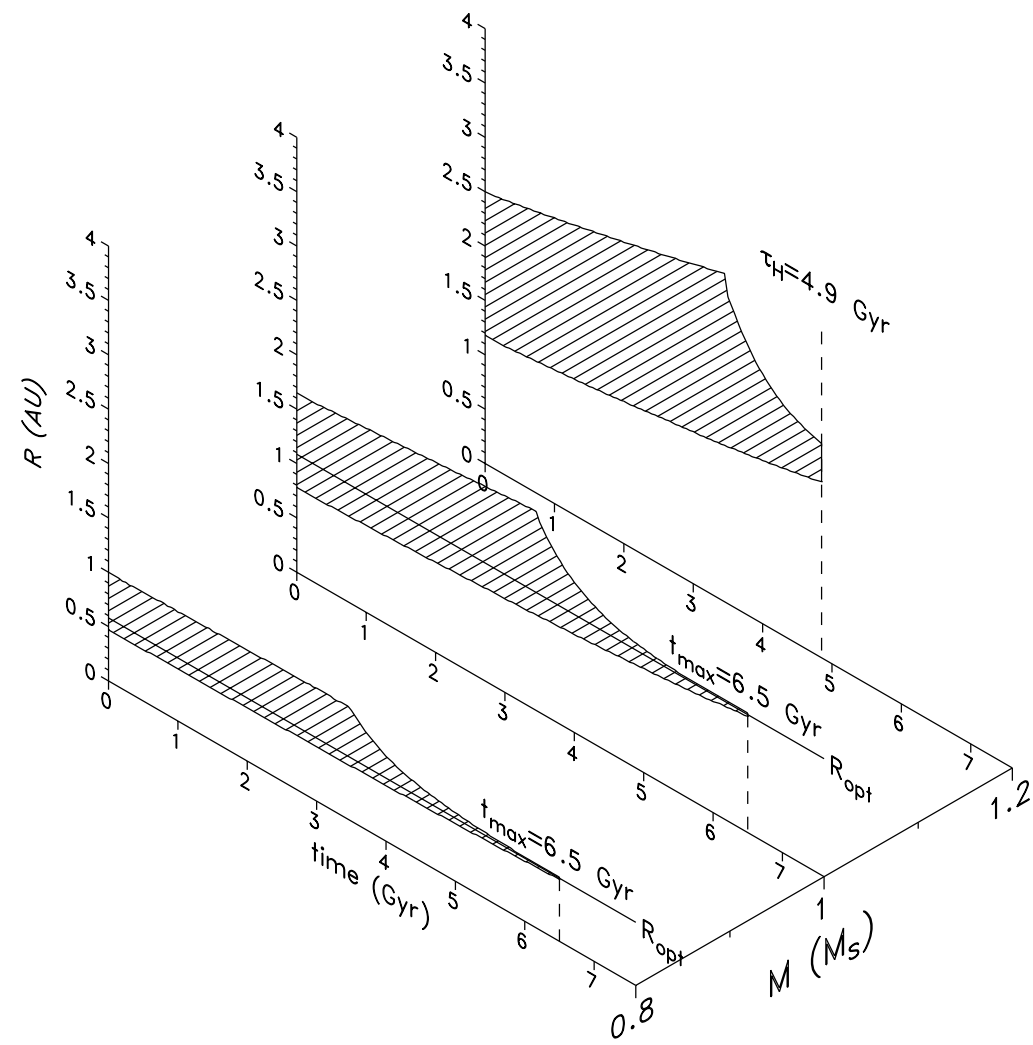

Fig 1. Width and position of the HZ (shaded) as a function of time for three different central-star masses $(\mathrm{M}=0.8,1.0,1.2$ $\mathrm{M}_{\mathrm{s}}$ ) for an Earth-like planet. $R_{\text {opt }}$ is the optimum position with maximum life span of the photosynthesis-based biosphere. 


\section{RESULTS AND DISCUSSION}

We can calculate the limits of photosynthesis in extrasolar planetary systems for any value of the central star mass between 0.2 and $2.5 \mathrm{M}_{\mathrm{s}}$. In Figure 1 we have plotted the width and position of the $\mathrm{HZ}$ for our geodynamic model (Franck et al., 1999, 2000a, 2000b) for three different central star masses $M=0.8,1.0$, $1.2 \mathrm{M}_{\mathrm{s}}$ over time. First we can find that the width and the position of the HZ depend strongly on the mass of the central star. Furthermore, up to about 3.5 Gyr of cogenetic stellar and planetary evolution the outer boundary of the $\mathrm{HZ}$ is steadily increasing as a result of increasing central-star luminosity. After this point, the continental area has grown to such a size that weathering is very effective in bringing $\mathrm{CO}_{2}$ out of the atmosphere and decreasing the outer boundary of the $\mathrm{HZ}$ which finally joins the inner one. For $1.2 \mathrm{M}_{\mathrm{s}}$ central stars the $\mathrm{C}_{4}$-type photosynthesis would be limited to $4.9 \mathrm{Gyr}$ after starting cogenetic evolution because the central star leaves the main sequence and becomes a red giant. For 0.8 and $1.0 \mathrm{M}_{\mathrm{s}}$ central stars the $\mathrm{C}_{4^{-}}$ type photosynthesis would be limited up to $6.5 \mathrm{Gyr}$ after starting cogenetic evolution because continental growth and decline in spreading rate force atmospheric $\mathrm{CO}_{2}$ content below $10^{-5}$ bar. In these cases, there is an optimum position $R_{\text {opt }}$ with maximum biospheric life span.

An alternative way to present our results about the limits of photosynthesis in extrasolar planetary systems is to delineate the HZ for an Earth-like extrasolar planet at a given (but arbitrary) distance in the stellar mass-time plane (see Figure 2). In this presentation the limits for photosynthesis are connected with the following effects (Franck et al., 2000b):

(I) Stellar life time on the main sequence decreases strongly with mass. We estimated the central hydrogen burning period and got $\tau_{H}<0.8 \mathrm{Gyr}$ for $M>2.2 \mathrm{M}_{\mathrm{s}}$. Therefore, there is no point in considering central stars with masses larger than $2.2 \mathrm{M}_{\mathrm{s}}$ because an Earth-like planet may need about 0.8 Gyr of habitable conditions for the development of life (Hart, 1978, 1979). Quite recently, smaller numbers for the time span required for the emergence of life have been discussed, for instance 0.5 Gyr (Jakosky, 1998). If we perform calculations with $\tau_{H}<0.5$, we obtain qualitatively similar results but the upper bound of central-star masses is shifted to $2.6 \mathrm{M}_{\mathrm{s}}$.

(II) When a star leaves the main sequence to turn into a red giant, there clearly remains no $\mathrm{HZ}$ for an Earth-like planet. This limitation is relevant for stellar masses in the range between 1.1 and $2.2 \mathrm{M}_{\mathrm{s}}$.

(III) In the stellar mass range between 0.6 and $1.1 \mathrm{M}_{\mathrm{s}}$ the maximum life span of the biosphere is determined by planetary geodynamics which is independent (in a first approximation, but see limiting effect 4) of $R$. So we obtain the limitation $t<t_{\max }$.

(IV) There have been discussions about the habitability of tidally locked planets. We take this complication into account and indicate the domain where an Earth-like planet on a circular orbit experiences tidal locking (Peale, 1977; Kasting et al., 1993; Joshi et al., 1997). That domain consists of the set of $(M, t)$ couples which generate an outer HZ-boundary below the tidal-locking radius. This limitation is relevant for $M$ $<0.6 \mathrm{M}_{\mathrm{s}}$.

In Figure 2 we depict the $\mathrm{HZ}$ for $R=2 \mathrm{AU}$. Under these circumstances, the limits of photosynthesis are given by stellar masses below $1.1 \mathrm{M}_{\mathrm{s}}$ and above $1.5 \mathrm{M}_{\mathrm{s}}$, and to stellar ages above $4.7 \mathrm{Gyr}$. 


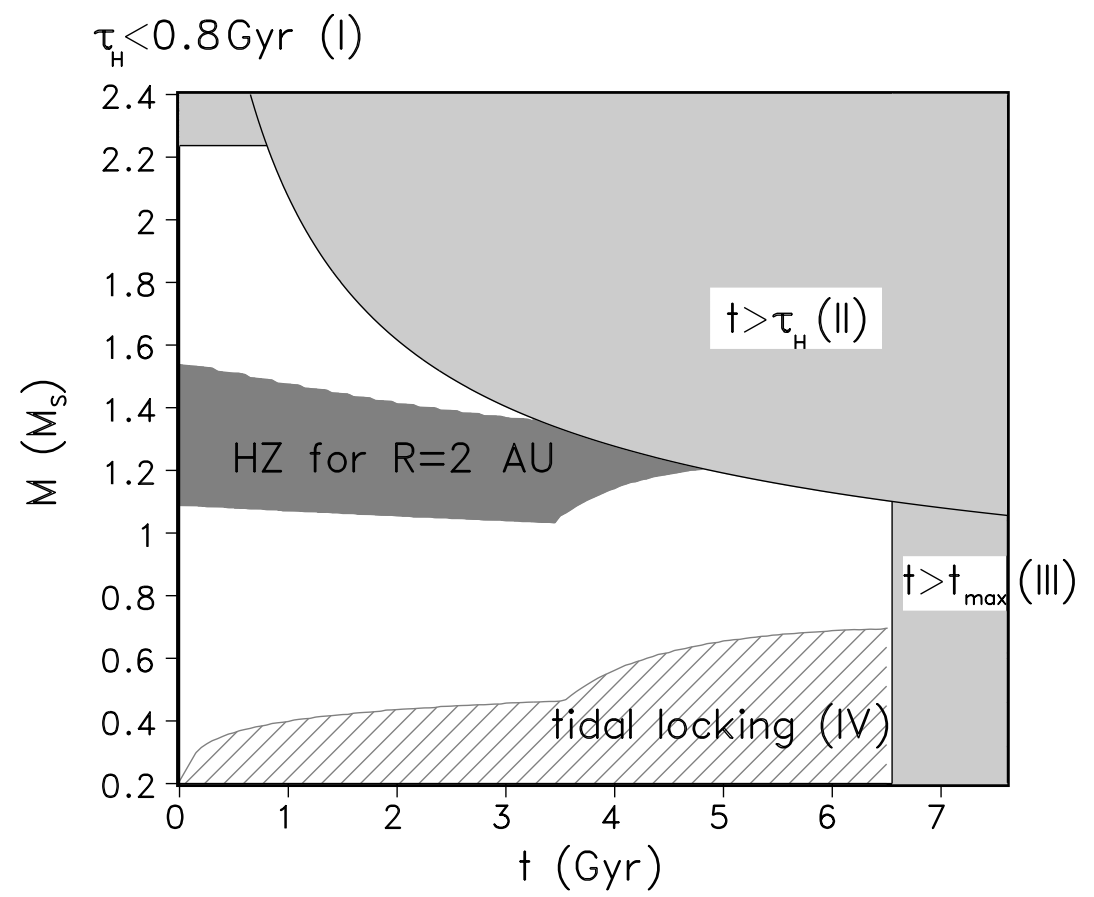

Fig 2. Shape of the HZ (dark grey) in the mass-time plane for an Earth-like planet with photosynthesis at distance $R=2$ AU from the central star. The potential overall domain for accommodating the $\mathrm{HZ}$ of planets at some arbitrary distance is limited by a number of $R$-independent factors that are explained in detail in the text. Figure is taken with slight changes from Franck et al., 2000b, copyright by the American Geophysical Union.

As we have shown above, our model provides a convenient filter for picking candidates for photosynthesis-based life from all the extrasolar planets that will be discovered in the future. Knowing the age and the mass of the central star and the distance between the star and an Earth-like planet one can decide whether the planet is habitable for photosynthesis-based life or not.

\section{REFERENCES}

Caldeira, K., and J. F. Kasting, The life span of the biosphere revisited, Nature, 360, 721-723,1992.

Doyle, L. R., Circumstellar Habitable Zones, Proceedings of the First International Conference, Travis House, 525 pp., Menlo Park,1996.

Franck, S., A. Block, W. von Bloh, C. Bounama, H. J. Schellnhuber, and Y. Svirezhev, Reduction of biosphere life span as a consequence of geodynamics, Tellus, 52B, 94-107, 2000a.

Franck, S., W. von Bloh, C. Bounama, M. Steffen, D. Schönberner, and H. J. Schellnhuber, Determination of habitable zones in extrasolar planetary systems: Where are Gaia's sisters?, J. Geophys. Res., 105(E1), 1651-1658, 2000b.

Franck, S., K. Kossacki, and C. Bounama, Modelling the global carbon cycle for the past and future evolution of the Earth system, Chem. Geol., 159, 305-317, 1999.

Hart, M. H., The evolution of the atmosphere of the Earth, Icarus, 33, 23-39, 1978.

Hart, M. H., Habitable zones around main sequence stars, Icarus, 37, 351-357, 1979.

Huang, S.-S., Occurrence of life in the universe, Am. Sci., 47, 397-402, 1959.

Huang, S.-S., Life outside the solar system, Sci. Am., 202(4), 55-63, 1960. 
Jakosky, B., The Search for Life on Other Planets, Cambridge Univ. Press, 326 pp., New York, 1998.

Joshi, M. M., Haberle, R. M., and Reynolds, R.T., Simulations of the atmsophere of synchronously rotating terrestrial planets orbiting $\mathrm{M}$ dwarfs: Conditions for atmospheric collapse and the implications for habitability, Icarus, 129(2), 450-465, 1997.

Kasting, J. F., Habitable zones around low mass stars and the search for extraterrestrial life, Origins Life, 27, 291-307, 1997.

Kasting, J. F., and T. P. Ackerman, Climatic consequences of very high carbon dioxide levels in the Earth's early atmosphere, Science, 234, 1383-1385, 1986.

Kasting, J. F., O. B. Toon, and J. B. Pollack, How climate evolved on the terrestrial planets, Sci. Am., 258(2), 46-53, 1988.

Kasting, J. F., D. P. Whitmire, and R. T. reynolds, Habitable zones around main sequence stars, Icarus, 101, 108-128, 1993.

Kippenhahn, R., and A. Weigert, Stellar Structure and Evolution, Springer-Verlag, 468 pp., New York, 1990.

Larcher, W., Physiological Plant Ecology: Ecophysiology of Functional Groups, Springer-Verlag, 506 pp., New York, 1995.

Peale, S.J., Rotation histories of the natural satellites, in Planetary Satellites, ed. J. A. Burns, pp. 87-112, Univ. of Ariz. Press, Tucson, 1977.

Pearcy, R. W., and J. Ehleringer, Comparative ecophysiology of $\mathrm{C}_{3}$ and $\mathrm{C}_{4}$ plants, Plant Cell Environ., 7, 113, 1984.

Schneider, S. H., and P. J. Boston, Scientists on Gaia, MIT Press, pp. 550, Cambridge, 1991.

Schwartzman, D. W., and T. Volk, Biotic enhancement of weathering and the habitability of earth, Nature, 340, 457-460, 1989.

Shklovskii, I.S., and C. Sagan, Intelligent Life in the Universe, Holden-Day, 509 pp., Merrifield, 1966.

Tajika, E., and T. Matsui, Evolution of terrestrial proto- $\mathrm{CO}_{2}$ atmosphere coupled with thermal history of the Earth, Earth Planet. Sci. Lett., 113, 251-266, 1992.

Volk, T., Feedbacks between weathering and atmospheric $\mathrm{CO}_{2}$ over the last 100 million years. Am. J. Sci., 287, 763-779, 1987.

Walker, J. C. G., P. B. Hays, and J. F. Kasting, A negative feedback mechanism for the log-term stabilization of Earth's surface temperature, J. Geophys. Res., 86, 9776-9782, 1981.

Williams, D. M., The stability of habitable planetary environments, Ph.D. thesis, Pa. State Univ., State College, 140 pp., 1998. 